Eco matemático ISSN 1794-8321 E-ISSN 2462-8794 Volumen 8 (s1) 2017, páginas 6 -15

\title{
El aprendizaje del cálculo y nuevas tendencias en su enseñanza en el aula de matemáticas
}

\author{
Learning calculus and new trends in his teaching in the math classroom \\ Cálculo de aprendizagem e novas tendências em seu ensino na sala de aula de matemática \\ Fernando Hitt ${ }^{\text {a* }}$ \\ ${ }^{a *}$ Doctor en Ciencias, Universidad de Montreal. Cánada.([)
}

\begin{abstract}
Forma de citar: Hitt, F. (2016). El Aprendizaje del cálculo y nuevas tendencias en su enseñanza en el aula de matemáticas. Encuentro Internacional en Educación Matematica ISSN 2539-1885. La Educación Matematica como Herramienta en el Desempeño Profesional Docente. Cúcuta, Colombia. 6 - 15.
\end{abstract}

Resumen: Los problemas de aprendizaje del cálculo han sido documentados desde hace varias décadas por los investigadores en didáctica de las matemáticas. Un gran esfuerzo en ese sentido lo ha proporcionado el grupo llamado "pensamiento matemático avanzado". Si bien el grupo ha propiciado una gran perspectiva sobre los problemas de aprendizaje del cálculo, el problema persiste del lado de la enseñanza. Entre los cambios realizados en el currículum promovidos por la investigación en didáctica del cálculo se pueden detectar tres grandes variantes. Una, ligada al impulso de las teorías sobre representaciones, que ha enfatizado los procesos de conversión entre representaciones, otra, ligada a métodos de enseñanza incluyendo tecnología; y una, más reciente, que enfatiza los procesos dela modelación matemática. Esta última, se perfila en la enseñanza del cálculo introduciendo situaciones problema (situaciones paradigmáticas) que tengan que ver directamente con la modelación matemática y uso de tecnología.

Palabras clave Aprendizaje, Cálculo, Enseñanza, Modelación matemática, Problema.

Abstract: The learning problems of calculus have been documented for several decades by researchers in mathematics didactics. A great effort in that sense has been provided by the group called "advanced mathematical thinking". Although the group has provided a great perspective on the learning problems of calculus, the problem persists on the teaching side. Among the changes made in the curriculum promoted by the research in didactics of the calculation can be detected three major variants. One, linked to the impulse of theories about representations, which has emphasized the conversion processes between representations, another, linked to teaching methods including technology; and one, more recent, that emphasizes the processes of mathematical modeling. The latter is outlined in the teaching of calculus introducing problem situations (paradigmatic situations) that have to do directly with mathematical modeling and use of technology.

Keywords Learning, Calculation, Teaching, Mathematical modeling, Problem.

Resumo: Os problemas de aprendizagem do cálculo foram documentados por várias décadas por pesquisadores em didática da matemática. Um grande esforço nesse sentido foi fornecido pelo grupo chamado "pensamento matemático avançado". Embora o grupo forneça uma grande perspectiva sobre os problemas de aprendizagem do cálculo, o problema persiste no lado do ensino. Entre as mudanças feitas no currículo promovido pela pesquisa em didática do cálculo podem ser detectadas três grandes variantes. Um, ligado ao impulso de teorias sobre representações, que tem enfatizado os processos de conversão entre representações, outro, ligado a métodos de ensino incluindo tecnologia; e um, mais recente, que enfatiza os processos de modelagem matemática. Este último é delineado no ensino de cálculo introduzindo situações-problema (situações paradigmáticas) que têm a ver diretamente com modelagem matemática e uso de tecnologia.

Palavras chave Aprendizagem, Cálculo, Ensino, Modelagem matemática, Problema.

* Autor para correspondencia fernando.hitt@gmail.com

2590-9215@ 2017 Universidad Francisco de Paula Santander. Este es un artículo bajo la licencia CCBY 


\section{Introducción}

El grupo denominado "Pensamiento avanzado" tuvo muchas repercusiones tanto en la reflexión sobre los problemas de aprendizaje de las matemáticas universitarias como en la implementación de nuevas experimentaciones en el aula. Este grupo de orden internacional tuvo un primer impacto con el libro editado por Tall (1991), "Advanced mathematical thinking". En este libro, los autores se enfocan más a los problemas del aprendizaje del cálculo que a su enseñanza. Si miramos de manera particular los cambios realizados en los Estados Unidos, podemos contar con más elementos de análisis.

En 1986 hubo un congreso en la Universidad de Tuluane en los Estados Unidos, y se considera que a partir de este congreso se inició en los Estados Unidos la Reforma del Cálculo. En esa época, como en el resto del mundo, los estudios en los Estados Unidos se centraron en los problemas de aprendizaje de los alumnos. Un artículo que causó mucho revuelo fue el de Selden, Mason \& Selden (1989). El ese estudio, Selden et al. (Idem), propusieron 5 problemas no rutinarios de cálculo, que se administró a una populación de estudiantes de ingeniería que ya habían aprobado el curso de cálculo con calificaciones regulares (no las mejores). En sus resultados se puede leer: Not one student got an entire correct.

En la década de los noventas, el curriculum de matemáticas, en lo general, inició un cambio substancial, la visualización matemática se consideró como importante en el aprendizaje de las matemáticas.Así, por ejemplo, Zimmermann \& Cunningham (1991) expresan que: Mathematical visualization is the process of forming images (mentally, or with pencil and paper, or with the aid of technology) and using such images effectively for mathematical discovery and understanding (p. 3), proporcionando a la noción de representación un lugar especial. Al mismo tiempo, la tecnología (las calculadoras y computadoras) emergía como una herramienta fundamental del aprendizaje al proporcionar en pantalla diferentes representaciones de un concepto matemático.

Todo ello dio pie al surgimiento de nuevos libros de texto. En ellos se puede apreciar que se eliminó la introducción al análisis en los reales (sucesiones y series) y se empezó a proporcionar más énfasis en el concepto de límite de funciones y al uso de tres representaciones: tabular, gráfica y algebraica. Bajo este enfoque tenemos, por ejemplo, el libro de Finney et al. (1994): Calculus. Graphical, Numerical, Algebraic. Este enfoque pretendía resolver los problemas de aprendizaje del cálculo que en el pasado su enseñanza era de corte puramente algebraica. Sin embargo, los problemas empezaron a emerger.
Si bien la tecnología proporcionaba diferentes representaciones de objetos matemáticos, la conversión entre representaciones no era una tarea fácil.

En esta misma época se formalizaron teorías sobre el aprendizaje centradas en las representaciones, por ejemplo, la de Duval (1995).

Así, al decidir que un aprendizaje conceptual del cálculo era necesario, implicaba el entender los problemas de los estudiantes en los procesos de conversión entre representaciones.

Cambios en el syllabus de cálculo y el rol del profesor de matemáticas El problema del aprendizaje del cálculo, quedó más claro al inicio de la década de los noventas. Se señalaba que habría que resolver los problemas ligados a los antecedentes al cálculo, y luego atacar los conceptos ligados al cálculo (Hitt 2013).

Tabla 1 Distinción entre antecedentes y conceptos propios al cálculo

\begin{tabular}{ll}
\hline \multicolumn{1}{c}{ Antecedentes al cálculo } & Conceptos propios del cálculo \\
\hline $\begin{array}{l}\text { Aritmética, álgebra, trigonometría, } \\
\text { geometría, geometría analítica. }\end{array}$ & $\begin{array}{l}\text { Límite, tangente, infinito potencial, } \\
\text { infinito actual, procesos al límite. }\end{array}$ \\
\hline
\end{tabular}

Esta distinción trajo consigo la distinción curricular entre pre-cálculo y cálculo, y la producción de libros ligados a cada uno de ellos. En uno de los estudios de ICMI (International Comission of Mathematics Instruction) (1986) propone que en un análisis curricular se deben contemplar tres aspectos:

a) Las intenciones de currículo.

b) El currículo implementado.

c) Logros del currículo.

En los párrafos anteriores nos hemos concentrado en los aspectos a) y c) de esta propuesta de ICMI. El punto b) es crucial en todo cambio curricular.

En los profesores recae un peso enorme, ya que entre autoridades educativas y editoriales con nuevos libros ponen al profesor en una situación difícil para el cambio. Ello es notorio en los estudios sobre el quehacer del profesor de matemáticas.

Para ejemplificar esta situación, tomemos una investigación realizada en Canadá en un instituto CEGEP, en donde se imparte el primer curso de cálculo. Hardy (2009a, 2099b) realiza un análisis en un Cégep sobre el concepto de límite.

Ella analiza lo que los profesores evalúan durante varios años y se encuentra que los exámenes ligados al concepto de límite son puramente algebraicos sin cuestionar diferentes representaciones de ese concepto. 
Tabla 2. Estudio de Hardy sobre la evaluación utilizada por los profesores sobre límites

\begin{tabular}{|c|c|}
\hline $\lim _{x \rightarrow 3} \frac{x^{3}-x^{2}-3 x-9}{2 x^{2}-4 x-6}$ & $\lim _{x \rightarrow 1} \frac{x^{2}-1}{(x-1)(x-3)}$ \\
\hline $\lim _{x \rightarrow 0} \frac{\sqrt{x+2}-\sqrt{2}}{x}$ & $\lim _{x \rightarrow \infty} \frac{9 x^{3}-x+2}{3 x^{3}+1}$ \\
\hline $\lim _{x \rightarrow \infty} \frac{x^{2}-25}{x^{3}-1}$ & $\lim _{x \rightarrow \infty} 7$ \\
\hline $\lim _{x \rightarrow 5} 3$ & $\lim _{x \rightarrow 1} 4 x^{3}+7 x-9$ \\
\hline $\lim _{x \rightarrow 2} \frac{x^{2}-4}{x^{2}-3 x-4}$ & $\lim _{x \rightarrow 4} \frac{\sqrt{x}-2}{x+4}$ \\
\hline $\lim _{x \rightarrow \infty} \frac{x^{3}+2 x+1}{x^{2}+7 x-1}$ & $\lim _{x \rightarrow \infty} \frac{x^{2}-1}{x-1}$ \\
\hline $\lim _{x \rightarrow \infty} \frac{\sqrt{2 x^{2}+1}}{3 x-5}$ & $\lim _{x \rightarrow \infty} \sqrt{x^{2}+1}-x$ \\
\hline $\lim _{x \rightarrow 1} \frac{x^{2}-x-2}{x-1}$ & $\lim _{x \rightarrow 1} \frac{x^{2}+6 x+19}{x^{3}-3 x+2}$ \\
\hline $\lim _{x \rightarrow 5} \frac{x^{2}-4}{x^{2}-25}$ & $\lim _{x \rightarrow 5} \frac{\sqrt{x+20}-\sqrt{5}}{5-x}$ \\
\hline
\end{tabular}

El estudio de Hardy muestra que los profesores evalúan diferentes tipos de técnicas para el cálculo de límites. Por ejemplo, podríamos agrupar las diferentes preguntas de acuerdo a la técnica a utilizar para calcular el límite.

a) Técnica de factorización, simplificación y paso al límite.

b) Técnica de multiplicar por el conjugado y paso al límite.

c) Técnica de factorización del término de grado más alto en la expresión algebraica, y paso al límite.

d) Reconocimiento de una función continúa en el punto que se desea calcular el límite, y paso al límite.

Uno se puede preguntar sobre los cambios en los últimos 10 años. En el caso de Quebec, la enseñanza pre-universitaria llega hasta los 18 años. El curso de cálculo es obligatorio para aquellos que quieren realizar estudios de ingeniería o ciencias.

En el 2001 en primaria (de 6 años) y 2004 en secundaria (de 5 años) se realizó un cambio en la enseñanza siguiéndose un acercamiento por competencias. La primera competencia de la disciplina en matemáticas es la resolución de situaciones problema. Esto aunado con los cambios en otros países, la modelación matemática llegó muy fuerte en los libros de cálculo.
El estudio de Hardy muestra que aún y los cambios en los syllabus y libros de texto en donde se enfatiza la importancia de la conversión entre representaciones, los exámenes se centran en el cálculo algebraico de límites. Los profesores no se han percatado de la importancia del rol de las representaciones en el aprendizaje de las matemáticas, cuando otro cambio les cae encima...en el 2008 recibieron la primera generación de estudiantes que se formaron en primaria y secundaria bajo un acercamiento por competencias y resolución de situaciones problema.

En este nivel de estudios preuniversitario, a diferencia de los otros niveles educativos previos, los syllabus no cambiaron fundamentalmente, pero los libros sí. Por ejemplo, un libro de texto (Hamel \& Hamiotte 2007) en el que sus dos primeras páginas las dedica a la historia de las matemáticas menciona el trabajo de Weirestrass sobre una función continua y no derivable en un punto:

$\boldsymbol{f}(\boldsymbol{x})=\sum_{\mathrm{n}=0}^{\infty} \boldsymbol{a}^{n} \cos \left(\boldsymbol{\pi} \boldsymbol{b}^{n} \mathrm{x}\right), 0<\mathrm{a}<1, \mathrm{~b}$ entero impar mayor a 1.

\section{¿Por qué iniciar con el trabajo de Weirestrass un libro de cálculo dirigido a estudiantes que por primera vez se enfrentan al infinito matemático?}

Los autores Hamel et Amiotte después de la parte histórica inician con un ejemplo de cálculo de límites asociado al llenado de un recipiente. Inmediatamente después con el lanzamiento de una pelota al aire en donde se proporciona la función ligada a un polinomio de $2^{\circ}$ grado y el cálculo de la velocidad instantánea en un momento dado.

Esto lo mencionamos para mostrar que los nuevos libros de texto tienen una nueva influencia ligada a la modelación matemática. No se sabe a ciencia cierta si el cambio se debe a este nuevo acercamiento por competencias y resolución de situaciones problema, al cambio que están sufriendo los libros en los Estados Unidos sobre modelación matemática o a ambos. Lo cierto es que el mundo académico está cada vez más comprometido con acercamientos ligados a la modelación matemática. Ver por ejemplo proyecto PRIMAS en Europa (Katja et al. 2013) y las producciones de ICME sobre la modelación matemática (p.e. Blum et al. 2007).

Nuevamente, los estudios en didáctica de las matemáticas nos señalan que los cambios en los libros de texto de cálculo en Quebec tienen poco impacto en la enseñanza. Por ejemplo, Dufour (2011) muestra cómo dos profesoras (una con 30 años de experiencia y otra con dos) utilizan los nuevos libros de cálculo en el aula de matemáticas. Precisamente, las dos profesoras utilizan el libro de texto de Hamel y Amiotte (Idem). Las dos inician la enseñanza de la derivada con el ejemplo del lanzamiento de una pelota al aire y el cálculo de la velocidad instantánea. 
El libro propone el siguiente ejemplo:

; Ejemplo $\overline{1} . \overline{2}$ uno de los problemas de base del cálculo diferencial es el de velocidad instantánea. Consideremos la situación siguiente. Se lanza una bola hacia । arriba a partir de una altura de $1 m$ con una velocidad I inicial de $9.8 \mathrm{~m} / \mathrm{s}$. En virtud de las leyes de la fisica, la posición de la bola (altura medida en metros) $t s$ después del lanzamiento está dada por la función $s(t)$ $=-4.9 t 2+9.8 t+1$. Se quiere determinar la velocidad (instantánea) de la bola a $0.5 \mathrm{~s}$ después de su lanzamiento.

Calculemos primero la velocidad media de la bola entre 0.5 s y 1 s.

Vamos las representaciones utilizadas por las profesoras:
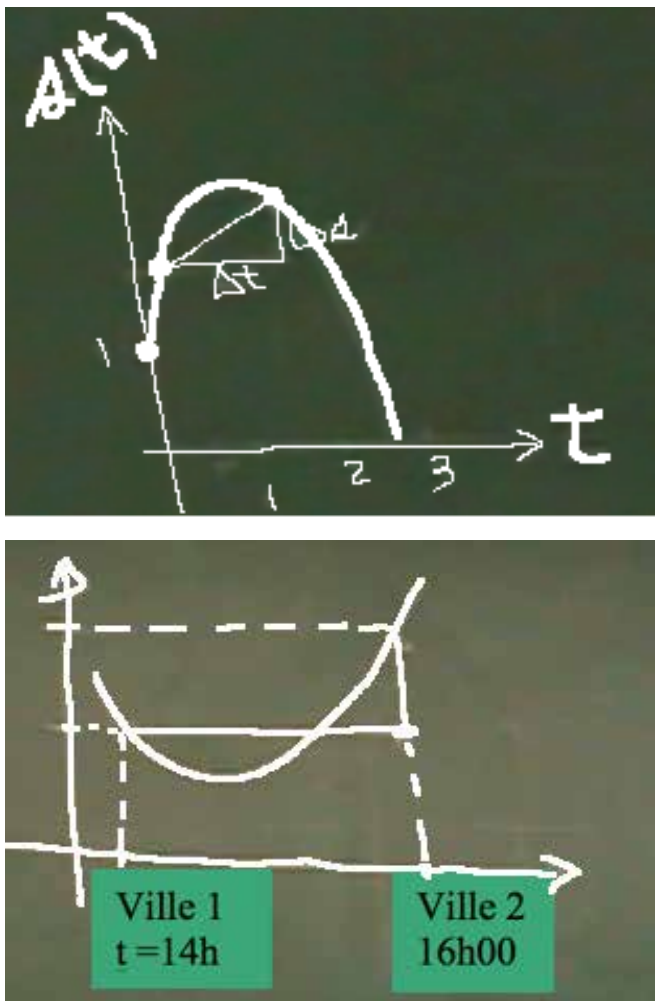

Fig. 1. Representaciones utilizadas por dos profesoras en la enseñanza de la derivada

\section{El libro propone el siguiente ejemplo:}

Las dos profesoras tienen una formación en matemáticas, la más joven con dos años de experiencia ha realizado estudios de maestría en educación general (no en didáctica de las matemáticas). La profesora con experiencia (30 años) utiliza la representación gráfica de la izquierda y la joven la de la derecha.

La profesora con experiencia lee el problema, pregunta si han entendido el fenómeno, hace la representación gráfica de la izquierda de la Figura 1, y pasa directamente al cálculo algebraico de la velocidad instantánea para $\mathrm{s}=1$ segundo.
La segunda profesora utiliza la misma representación (Figura 1 derecha) para modelar diferentes fenómenos: peso de un bebé al pasar el tiempo, viajar en auto de una ciudad a otra (cayendo en contradicción en sus interpretaciones).

En los dos casos, es notorio el aspecto pasajero de la representación. Hay que ir de inmediato a la manipulación algebraica. En el caso de la joven es peor, ya que la representación gráfica que utiliza no es adecuada para el fenómeno en estudio.

Con ello, lo que queremos enfatizar es que aún y cuando las teorías sobre la construcción del conocimiento, basadas en la conversión entre representaciones ha avanzado en los últimos años, estos aspectos teóricos no han sido del todo asimilados por el profesor de matemáticas. ¿Los profesores han recibido una actualización en ese sentido?

La tecnología y un método de enseñanza (ACODESA) Como lo hemos mencionado anteriormente, una carga enorme recae en el profesor de matemáticas en un cambio curricular. Sería importante que los investigadores en didáctica de las matemáticas fueran más sensibles a esta problemática.

Es en esta dirección en la que queremos ahondar en lo que sigue. La tecnología ha avanzado considerablemente, no solamente proporcionando diferentes representaciones de un concepto matemático en pantalla de calculadoras o computadoras. Existe desde hace varios años el uso de paquetes libres para análisis de datos de fenómenos físicos (por ejemplo, AVIMECA para PC, o Tracker para PC o Mac). Nosotros utilizaremos Tracker para mostrar un uso de este tipo de software.

Antes de mostrar Tracker con un ejemplo, queremos mostrar la diferencia que existe en los procesos clásicos de modelación y cómo los consideramos ahora.

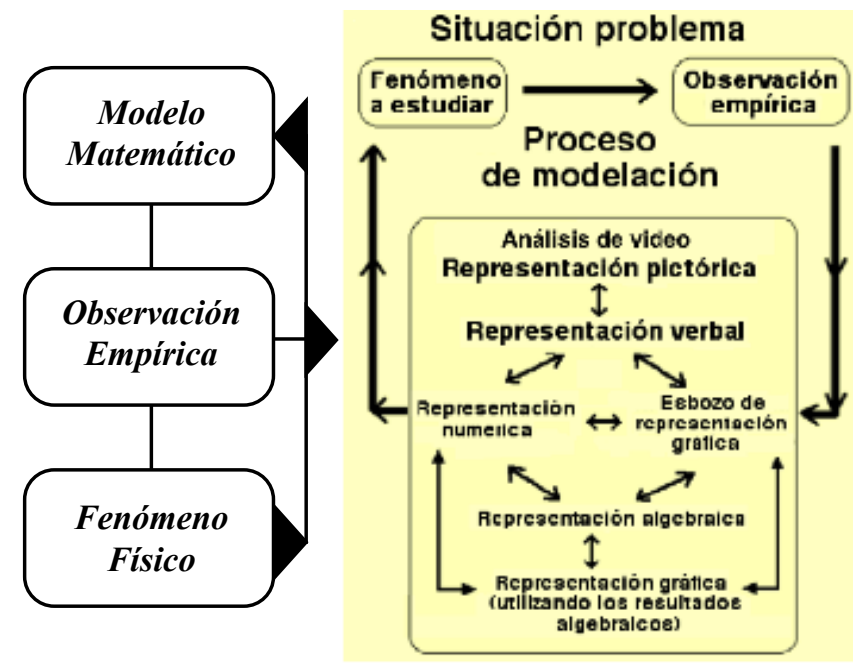

Fig. 2. Modelo clásico de la modelación y nuevas tendencias con el uso de videos 
En la Figura 2, mostramos un modelo clásico de proceso de modelación y un nuevo modelo adaptado a una teoría local de representaciones (ver Hitt \& Quiroz en prensa) y análisis de videos con una herramienta como Tracker. En este modelo, se hace un especial énfasis al solicitar a los estudiantes que proporcionen una descripción verbal de fenómeno en estudio identificando las variables que entran en juego.

Si se siguiera un método de enseñanza ligado a una teoría general sociocultural del aprendizaje (Hitt \& Quiroz Idem), la comunicación entre los alumnos y el profesor como guía, es el ingrediente principal en el aprendizaje de conceptos matemáticos.

Nuestro método de enseñanza de corte sociocultural, basado en un aprendizaje en colaboración, en debate científico y autoevaluación llamado ACODESA (siglas en francés Apprentissage Collaborative, Débat Scientifique et Autoréflexion) consta de 5 etapas (Hitt 2007; Hitt y González-Martín 2015, Hitt, Saboya y Cortés 2016, Hitt \& Quiroz Idem):

1) Trabajo individual (producción de representaciones funcionales y producciones semióticas asociadas para comprender la situación problema).

2) Trabajo en equipo sobre una misma situación. Proceso de discusión y validación (refinamiento de las representaciones funcionales y producciones asociadas).
3) Debate (que puede convertirse en un debate científico). Proceso de discusión y validación (refinamiento de representaciones funcionales y producciones asociadas).

4) Auto-reflexión. Regreso sobre la situación (trabajo individual de reconstrucción y auto-reflexión de lo realizado en clase).

5) Institucionalización. Utilización de representaciones institucionales por parte del profesor dentro del proceso de institucionalización de saberes.

Es importante señalar que, bajo este enfoque, el rol del profesor es diferente, en este caso, el profesor:

a) Diseña la tarea a realizar en el grupo, siguiendo las características del método ACODESA de enseñanza (situación que se realiza en varias etapas).

b) Guía a los alumnos durante las 4 primeras etapas de ACODESA, promoviendo la reflexión, argumentación, validación de las acciones de los alumnos, sin proporcionar respuestas si es correcto o no lo que los alumnos realizan.

c) Recopila y organiza a grandes rasgos las producciones de los alumnos, y proporciona el proceso de resolución institucional, utilizando las representaciones institucionales correspondientes.

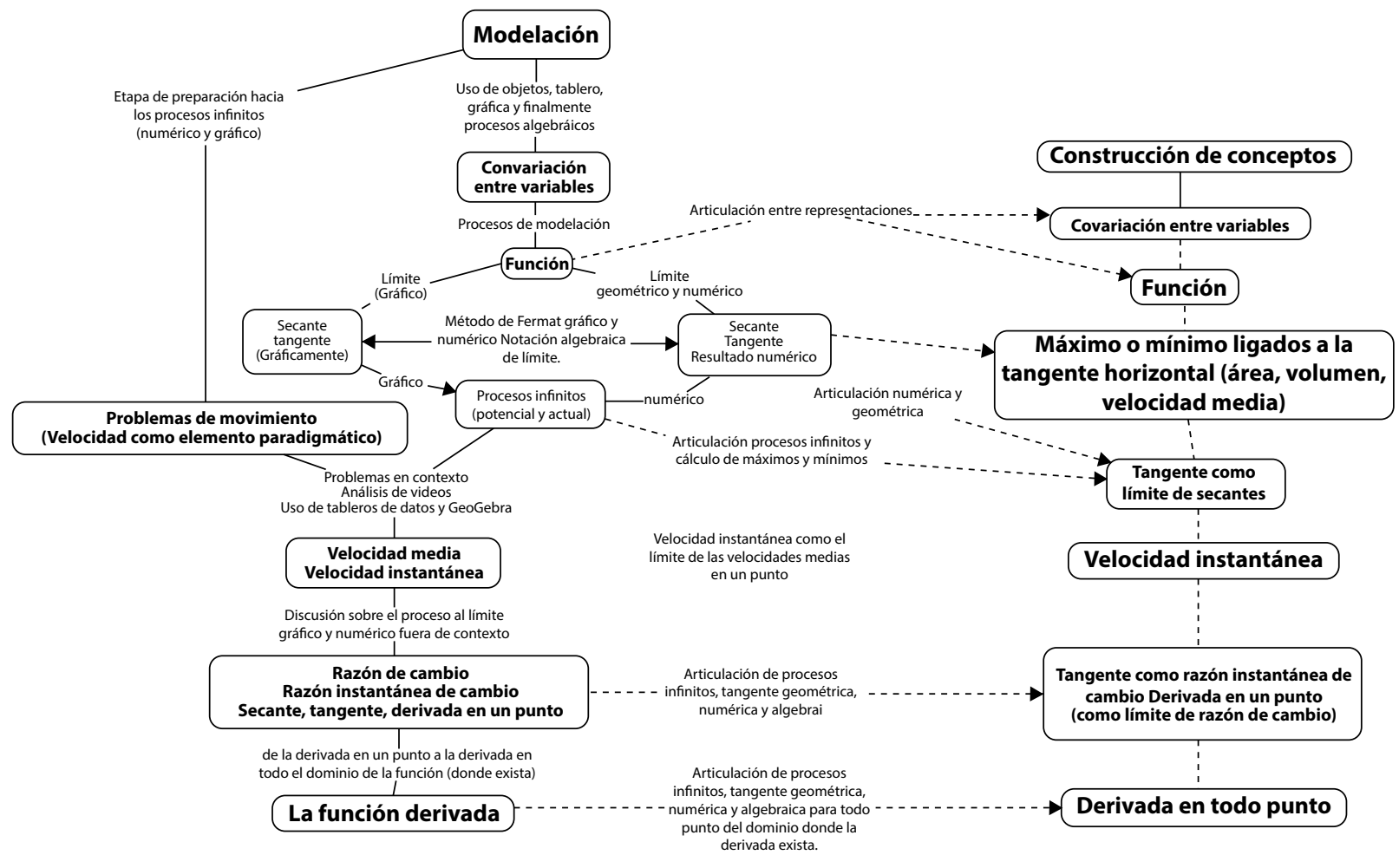

Figura 3. Una trayectoria global de aprendizaje (TGA) para la introducción al cálculo 
La tecnología y un método de enseñanza (ACODESA) y la investigación en apoyo al profesor de matemáticas La sección precedente, solicita de mucho trabajo de parte del profesor. Los libros de texto en general van en retraso con respecto a los avances tecnológicos, y muchos de esos libros son traducciones de libros que siguen métodos de enseñanza diferentes a los que el profesor desea implementar en su clase.

Recuerdo que mi primer libro de cálculo en la universidad fue el Kuratowski (1962), libro muy difícil para mí y mis compañeros. El segundo libro que me propusieron en el siguiente curso de cálculo fue el Spivak (1967). De este libro los profesores decían, es un libro magnífico por la selección de sus problemas. Con este libro, años después ya en didáctica de las matemáticas, comprendí la importancia de los procesos de visualización matemática y la resolución de problemas.

Con lo anterior, lo que quiero decir, es que el profesor de matemáticas necesita de problemas bien estructurados, que le permita proponer en su clase y promover un aprendizaje significativo. En nuestro caso, sería importante crear un banco de videos cortos, junto con una actividad y que el profesor pueda utilizarlos en su enseñanza.

Vamos a proporcionar un ejemplo. Supongamos que estamos interesados en una introducción al cálculo bajo este nuevo enfoque de la modelación matemática. Consideremos la siguiente trayectoria global de aprendizaje (adaptación de la idea que propone Simón 1995, Simón \& Tzur 2004, de su THA: Trayectoria Hipotética de Aprendizaje). Simon\& Tzur proponen que, para cada organización de enseñanza en el aula, es conveniente que el profesor realice una THA específica; en nuestro caso, el acercamiento es global en una primera instancia (Figura 3).

La literatura actual señala la importancia de la covariación entre variables como preludio al concepto de función (Hitt \& González-Martín, 2015, 2016; Thompson \& Carlson 2017). En nuestro caso, hemos propuesto una serie de actividades para trabajarse en ambientes de papel y lápiz con el método ACODESA y uso de tecnología de parte del profesor en la etapa de institucionalización. En este documento, queremos introducir el uso del video teniendo como meta el concepto de derivada.

De acuerdo a nuestra TGA (Figura 3), debemos pasar por una discusión de la velocidad media y de la velocidad instantánea.

El análisis de textos tanto de Quebec como el texto Harvard Calculus (Huges et al. 1999) de los Estados Unidos, introducen el concepto de derivada utilizando una actividad ligada a la física, el lanzamiento de una pelota en el caso de Quebec (Hamel \& Amiotte 2007) y el lanzamiento de una toronja en el caso del Harvard Calculus (Huges et al. 1999).
Como lo habíamos señalado antes, Dufour (2011) en su tesis de maestría, analizó dos profesoras en un curso de introducción al cálculo. Las profesoras mencionaban el fenómeno del lanzamiento de una bola al aire, y utilizaban un polinomio de $2^{\circ}$ grado proporcionado por el libro de texto, pasando directamente a los procesos algebraicos. Los estudiantes seguían una explicación sin argumentar de porqué se tendría que calcular velocidades medias y la velocidad instantánea. Otro hecho importante es que, aún con todos los cambios realizados en Estados Unidos sobre la reforma del cálculo: cambios en el currículo, nuevos libros de texto, cambio de cursos en auditorio a cursos con 25 alumnos con enseñanza de trabajo en colaboración; los problemas del aprendizaje del cálculo continúan vigentes (Star \& Smith 2006).

Nosotros proponemos la actividad de utilizar el concepto de velocidad (Hitt \& Dufour 2014), pero ya sea poniendo a alguien a utilizar una bicicleta o corriendo frente a una cámara (Figura 4).

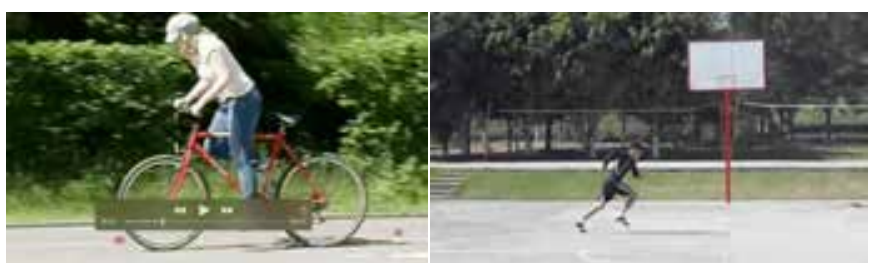

Figura 4. Cámara fija para la toma de datos, y medida donde se pasa la acción (señalamiento de $2 \mathrm{~m}$ por donde pasa la bicicleta, $15 \mathrm{~m}$ ancho de la cancha)

Una vez observando el video y selección de variables, lo deslizamos al interior de Tracker y ponemos en marcha el proceso para la toma de datos (ejes, distancia de la cancha de básquet, cuando iniciar y finalizar la toma de datos. En experimentaciones informales con los estudiantes, al proporcionarles la actividad (ver anexo), los estudiantes de manera natural proporcionan una lista de variables a analizar. Entre ellas, proponen la distancia recorrida en función del tiempo. Al cuestionarlos sobre qué preguntas se les ocurre proponer, siempre ha surgido de manera natural la pregunta: ¿En qué momento el corredor alcanza su velocidad máxima?.

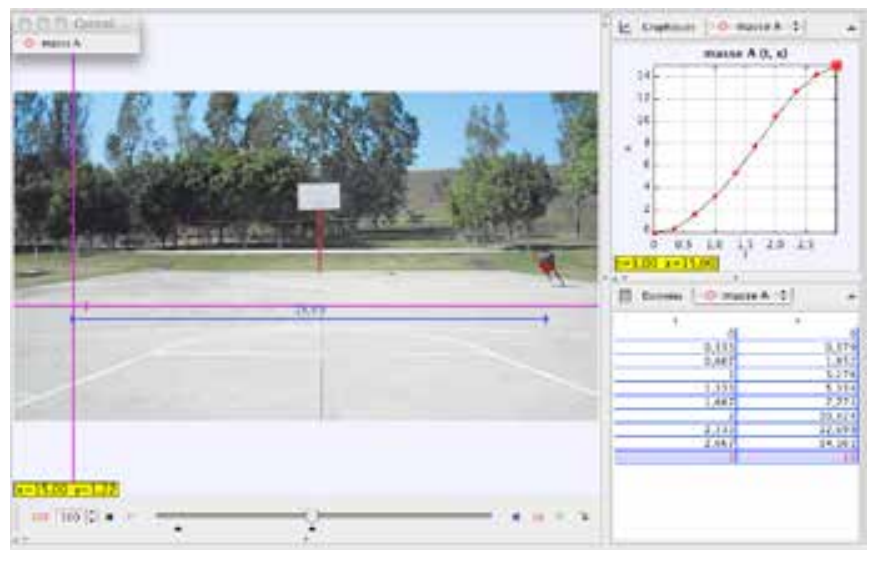

Figura 5. Toma de datos, distancia recorrida en función del tiempo 
Una vez tomados los datos con Tracker de acuerdo a las variables seleccionadas (esquina inferior derecha), uno podría optar por un análisis utilizando Tracker o los puede copiar directamente sobre otro paquete (libre) como el GeoGebra. Nosotros hemos seleccionado GeoGebra, ya que este software permite un tratamiento de datos flexible y en la resolución de la situación problema, es posible utilizar cálculo formal.
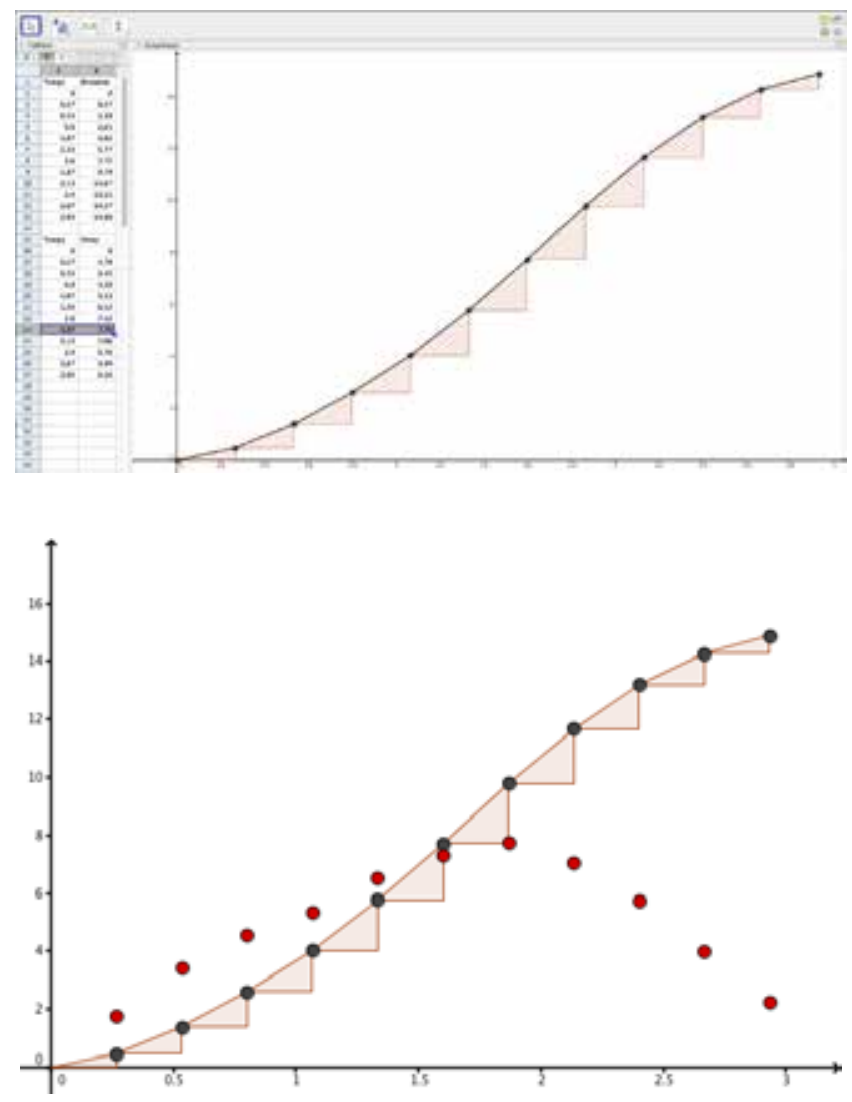

Figura 6. Tratamiento de datos con GeoGebra y cálculo de velocidades medias para cada intervalo de tiempo

Para el cálculo de la velocidad instantánea, y sabiendo que el fenómeno lo podemos modelar con una función continua, los estudiantes bajo una discusión sobre las características del modelo a utilizar, el cambio de concavidad emerge en su propuesta. En general, después de una discusión, proponen ya sea un polinomio de 3er grado $\mathrm{y} / \mathrm{o}$ una función sinusoidal.

Es posible tomar intervalos más cortos en Tracker, pero es importante que el profesor promueva la idea de paso al límite para calcular la velocidad instantánea (la noción de tangencia debe haberse trabajado antes). Una posible manera de contrastar las producciones de los alumnos es que el profesor utilice GeoGebra con alguna de las funciones propuestas (Figura 7) para realizar el paso al límite y proporcionar la definición de derivada después.

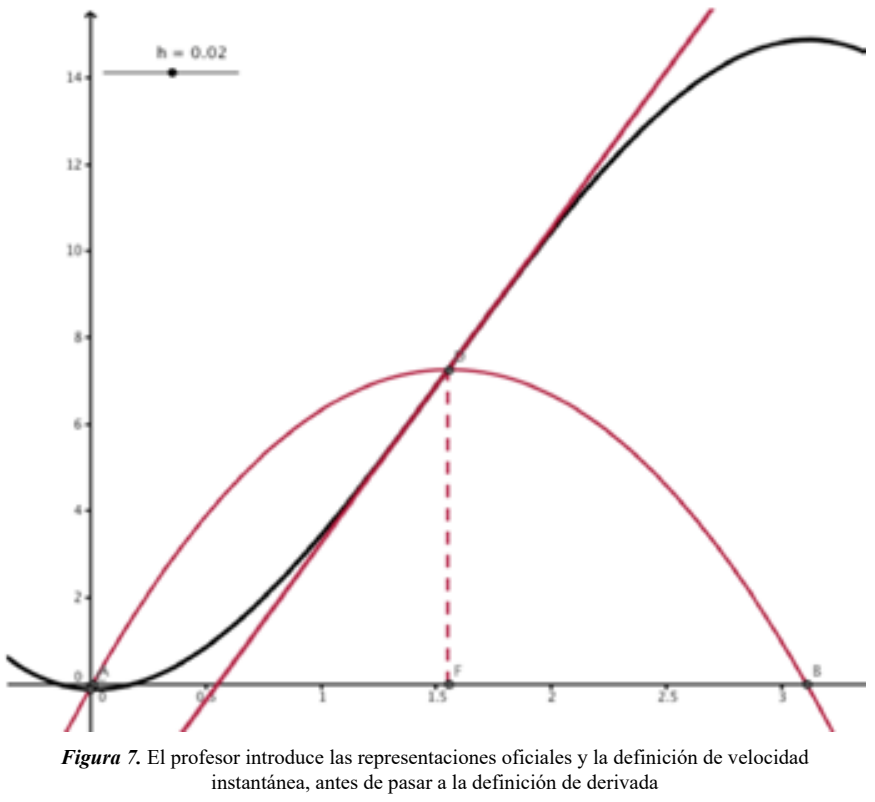

A manera de conclusión Los cambios curriculares apuntan hacia un mayor énfasis en los procesos de modelación matemática. Estos procesos de modelación no son exclusivos de la enseñanza universitaria. En 13 universidades europeas (proyecto PRIMAS, Katja et al. 2013) se ha impulsado la unificación de las didácticas (biología, física, matemáticas, química) poniendo la modelación matemática como elemento central. Bloom et al. (2007) proponen la modelación a diferentes niveles educativos.

En Quebec el acercamiento por competencias y resolución de situaciones problema ha puesto en dificultades a los profesores ya que el ministerio no tomó en cuenta la importancia de la modelación matemática bajo este acercamiento. De acuerdo al estudio de Zandieh (2000), en los Estados Unidos el concepto de velocidad es utilizado en el aula en la enseñanza del cálculo.

Esta tendencia en la enseñanza de las matemáticas a todos niveles, tendría un soporte importante con el uso de la tecnología en el aula de matemáticas. Sin embargo, como lo hemos dicho en este documento, es importante proporcionar al profesor de matemáticas con actividades y videos experimentados para que él haga uso de ellos en el aula.

Dejar una discusión totalmente libre en el aula puede promover un caos que a muchos estudiantes y profesores no les agrada. Por ello, el diseño de actividades ad hoc (ver APTE 2016 en el caso álgebra) es una tarea prioritaria para profesores e investigadores en didáctica de las matemáticas (Hitt, Saboya \& Cortés 2017). 


\section{Referencias}

APTE. (2016). Algèbre en partenariat avec la technologie en éducation. http://www.math.uqam.ca/ apte/Taches.html.

Blum, W., Galbraith, P., Henn, H. \& Niss, M. (Eds. 2007). Modelling and applications in mathematics education. The 14th ICMI Study. New York: Springer.

Dufour, S. (2011). L'utilisation des représentations par deux enseignants du collégial pour l'introduction de la dérivée. Mémoire de maîtrise. Université du Québec à Montréal. http://www.archipel.uqam.ca/4059/.

Duval, R. (1995). Sémiosis et pensée humaine: Registres sémiotiques et apprentissages intellectuels. Neuchâtel: Peter Lang.

Finney, R., Thomas, G., Demana, F. \& Waits, B. (1994). CALCULUS. Graphical, Numerical, Algebraic. De. Addison -Wesley.

GeoGebra (software libre: http://www.geogebra.org/cms/)

Hamel, J., \& Amyotte, L. (2007). Calcul différentiel (p. 449). Canada: Édition du Renouveau Pédagogique Inc.

Hardy, N. (2009a). Students' models of the knowledge to be learned about limits in college level calculus courses. The influence of routine tasks and the role played by institutional norms. Unpublishes thesis. Concordia University.

Hardy, N. (2009b). Students' perceptions of institutional practices: The case of limits of functions in college level Calculus courses. Educational Studies in Mathematics, 72(3), 341-358.

Hitt F. (2007). Utilisation de calculatrices symboliques dans le cadre d'une méthode d'apprentissage collaboratif, de débat scientifique et d'auto-réflexion. In M. Baron, D. Guin et L.Trouche (Éds.), Environnements informatisés et ressources numériques pour l'apprentissage. conception et usages, regards croisés (pp. 65-88). Paris: Hermès.

Hitt, F. (2013). El infinito en matemáticas y el aprendizaje del cálculo: Infinito potencial versus infinito real. Revista de Matemática Educativa del Cinvestav-IPN, El Cálculo y su Enseñanza, 4, 103-122. http://mattec.matedu.cinvestav.mx/el_calculo/.

Hitt, F. et Dufour, S. (2014). Un análisis sobre el concepto de derivada en el nivel preuniversitario, del rol de un libro de texto y su posible conexión con el uso de tecnología. In A. Cuevas \& Pluvinage F. (Eds.), La enseñanza del cálculo diferencial e integral (pp.19-42). México City: Pearson Educación.

Hitt, F. \& González-Martín, A.S. (2015). Covariation between variables in a modelling process: The ACODESA (Collaborative learning, Scientific debate and Self-reflexion) method. Educational Studies in Mathematics, 88(2), 201-219.
Hitt, F. \& González-Martín A. (2016). Generalization, covariation, functions and calculus. PME contributions in the last ten years. In A. Gutiérrez, G. Leder and P. Boero (Eds.), Handbook of Research on the Psychology of Mathematics Education (pp. 3-38). Rotterdam/Taipei: Sense.

Hitt, F., and Quiroz, S. (en prensa). Aprendizaje de las matemáticas a través de la modelación matemática en un medio sociocultural ligado a la teoría de la actividad. Revista Colombiana de Educación.

Hitt, F., Saboya, M. and Cortés C. (2017). Rupture or continuity: the arithmetic algebraic thinking as an alternative in a modelling process in a paper and pencil and technology environ nement. Educational Studies in Mathematics. Dordretch: Springer. DOI 10.1007 /s 10649-016-9717-4.

Hitt, F., Saboya, M. and Cortés C. (2017). Task design in a paper and pencil and technological environment to promote inclusive learning: An example with polygonal numbers. In G. Aldon, F. Hitt, L. Bazzini \& Gellert U. (Eds.), Mathematics and techn ology. A C.I.E.A.E.M. Sourcebook (pp. 57-74). Cham : Springer.

Howson, G. \& Wilson, B. (Eds.) (1986). School Mathematics in the 1990's. ICMI Study Series. Cambridge: Cambridge University Press.

Katja, M., Reitz-Koncebovski, K. \& Billy, G. (2013). Inquiry-based learning in maths and science classes. What it is and how it works-examples-experiences. Freiburg: EU \& U. of Education de Freiburg, Germany). http://www.primas-project.eu/fr/index.do.

Kuratowski, K. (1962). Introduction to calculus. Oxford: Oxford Pergamon Press.

Selden, J., Mason, A. \& Selden, A. (1989). Can Average Calculus Students Solve Nonroutine Problems? Journal of Mathematical Behavior 8, 45-50.

Simon, M. A. (1995). Reconstructing mathematics pedagogy from a constructivist perspective. Journal for Research in Mathematics Education, 26, 114-145.

Simon M. A. \& Tzur R. (2004), THA: Trayectoria Hipotética de Aprendizaje.

Star, J.R., \& Smith, J. (2006). An image of calculus reform: Students' experiences of Harvard calculus. Research in Collegiate Mathematics Education, 13, 1-25.

Tall, D. (1991) (Ed.). Advanced mathematical thinking. Dordrecht: Kluwer Academic Publishers.

Thompson, P. W., \& Carlson, M. P. (2017). Variation, covariation, and functions: Foundational ways of thinking mathematically. In J. Cai (Ed.), Compendium for research in mathematics education (pp. 421-456). Reston, VA: NCTM. 
Tracker. Logiciel libre. Video analysis and modeling tool (version 4.87). Reference, 8-janvier-2015. http://www.cabrillo.edu/ dbrown/tracker/.

Zandieh, M. (2000). A theoretical framework for analyzing student understanding of the concept of derivative. Research in Collegiate Mathematics Education, Vol. VIII, pp. 103-127.

Zimmermann, W. \& Cunningham, S. (Eds). (1991). Visualization in Teaching and Learning.19, USA: MAA Series.

De La Barrerar, \&Donolo. (2009). Neurociencias y su importancia en contextos de aprendizaje. Revista Digital Universitaria 10 de abril 2009, Recuperado de h t t p : / / w w w . revis ta.una m. mx/vol.10/n um4/art20/art20.pdf

De La Cruz, M. (2004). Distintos planteamientos sobre el problema cuerpo-mente. En J. Gómez, Neurociencia cognitiva y Educación (págs. 12-30). Lambaye que: Fondo Editorial FACHSE.

GAZZANIGA, M. (2002): CognitiveNeuroscience. Norton \& Co, Nueva York.

Hart, L. (1999). Cerebro Humano y Aprendizaje Humano. Libros para educadores.

Kandel, E., Schwartz, J., \&Jesell, T. (2001). Principios de neurociencia. Madrid: McGraw- Kandel, E. R. (1997). Neurociencia y conducta.

Martínez, M. (2004). Ciencia y arte en la metodología cualitativa. México: Trillas.

Mora, F. (2007). Neurocultura. Una cultura basada en el cerebro. Madrid: Alianza

Mora, Francisco (2013). Neuroeducación: sólo se puede aprender aquello que se ama. Alianza Editorial

Meltzer, L. (Ed.) (2007). Executive Function in Education. New York: Guilford Press.

Perea, M. V. (Abril, 2008). Rehabilitación cognitiva. Conferencia Inaugural del Doctorado en Neuropsicología, Universidad de Salamanca- Universidad Iberoamericana. Costa Rica: UNIBE

Punset, E. (2007). El alma está en el cerebro. Radiografía de la máquina de pensar. México: Santillana

Pizarro De Zulliger, B. (2003). Neurociencia y Educación

Revista Electrónica “Actualidades Investigativas en Educación” Volumen 5, número 1, año 2005.
Ruiz Bolívar, C. Neurociencia y Educación. Disponible en http://www.revistaparadigma.org.ve/doc/paradigma96/doc4.htm

Vygotski, L. S. (1995). Obras Escogidas III: Desarrollo de la Psique. (Trad. de Julio Guillermo Blank). Madrid: Visor. 


\section{Anexo}

\begin{tabular}{|c|c|}
\hline \multicolumn{2}{|c|}{ La competencia del siglo, Aquiles contra la tortuga } \\
\hline Nombre del equipo: & $\begin{array}{l}\text { Consignas: } \\
\text { Para el trabajo individual/inicial, utilizar } \\
\text { boligrafo de tinta negra. }\end{array}$ \\
\hline------- & $\begin{array}{l}\text { Para el trabajo en equipo (o especificar el } \\
\text { momento), si tu modificas tu trabajo, utilizar } \\
\text { un boligrafo de tinta roja. }\end{array}$ \\
\hline $\begin{array}{l}\text { Nombres de los } \\
\text { miembros del equipo: } \\
\end{array}$ & $\begin{array}{c}\text { Después del debate en gran grupo (o } \\
\text { especificar el momento), si tu modificas tu } \\
\text { trabajo, utilizar un boligrafo de tinta verde. } \\
\text { Aquiles contra la tortuga }\end{array}$ \\
\hline------- & \\
\hline------- & \\
\hline------- & \\
\hline-------- & \\
\hline
\end{tabular}

\begin{tabular}{|l|}
\hline \multicolumn{1}{|c|}{ Trabajo individual } \\
\hline \hline Consignas: \\
Aquiles está en constante preparación para la competencia contra la \\
tortuga. Ya que la competencia está programada en varias etapas, \\
Aquiles inicia su preparación con la carrera a pie. \\
Puedes observar el video para \\
responder a las preguntas \\
siguientes: \\
Preguntas globales: \\
a) ¿Cuáles son las variables que \\
debemos tomar en cuenta? \\
a) ¿Cuál es la velocidad media de \\
Aquiles al atravesar la cancha de \\
15 m?
\end{tabular}

\section{Trabajo en equipo}

Observando el video, ¿Cuáles son las variables que debemos tomar en cuenta? Explica y cuestiona tus repuestas con las de tus compañeros.

\section{Trabajo en equipo}

Utilizando Tracker, determinar la velocidad media al atravesar la cancha de un lado al otro.

Dato que puede ser útil para esta primera etapa con Tracker:

Ancho de la cancha de básquet: $15 \mathrm{~m}$.

\section{Trabajo en equipo}

Una vez que se han fijado las imágenes por segundo (por ejemplo 10 la la vez, 5 la segunda, etcétera). Calcular la velocidad media para cada intervalo con la ayuda de otro paquete como GeoGebra. Representar con una nube de puntos los datos obtenidos con Tracker. ¿Es posible representar el fenómeno con una curva continua? ¿Tienen alguna conjetura? Argumentar y justificar.

¿Es posible proporcionar una respuesta sobre en qué momento Aquiles alcanza su velocidad máxima? Argumentar, explicar, calcular, demostrar,...

Trabajo en equipo

Contrastar los diferentes resultados encontrados por los compañeros de otros equipos. Argumentar y justificar.

¿Es posible proporcionar una respuesta sobre en qué momento Aquiles alcanza su velocidad máxima? Argumentar, explicar, calcular, demostrar,...

\begin{tabular}{l} 
Regreso al trabajo individual, reconstrucción de lo realizado en clase \\
\hline \hline Considerar el video de la carrera de Aquiles de ida y vuelta. \\
Toma datos con Tracker de la distancia entre Aquiles y el punto de \\
partida. ¿Puedes modelar el fenómeno con una curva continua? \\
¿Es posible proporcionar una respuesta sobre en qué momento Aquiles \\
alcanza su velocidad máxima de ida y luego de regreso? ¿Alcanza la \\
misma velocidad máxima de ida que de vuelta? Argumentar, explicar, \\
calcular, demostrar,...
\end{tabular}

Etapa final de institucionalización de la parte del profesor. 